\title{
Nanotechnology Engulfing Dentistry
}

Chawla $\mathbf{R}^{1^{*}}$, Shetty $\mathrm{KS}^{2}$, Prakash $\mathrm{AT}^{2}$, Rathore APS ${ }^{2}$ and Saroch $\mathbf{S}^{3}$

${ }^{1}$ Department of Orthodontics, Penang International Dental College, Penang, Malaysia

${ }^{2}$ Department of Orthodontics and Dentofacial Orthopedics, Bapuji Dental College and Hospital, Davangere, Karnataka, India

${ }^{3}$ Amrit Scan and Dental Hospital, Hoshiarpur, Punjab, India

*Corresponding author: Dr. Ridhi Chawla, Department of Orthodontics, Penang International Dental College, Penang, Malaysia, Tel: +60162919468; E-mail: riddhichawla@yahoo.co.in

Received date: January 2, 2018; Accepted date: April 19, 2018; Published date: April 26, 2018

Copyright: (C) 2018 Chawla R, et al. This is an open-access article distributed under the terms of the Creative Commons Attribution License, which permits unrestricted use, distribution, and reproduction in any medium, provided the original author and source are credited.

\begin{abstract}
Nanoengineering is a mushrooming branch with which humans wish to create self-free capitulating creatures that walk around and even dream from mere inanimate ingredients. It has engulfed almost every field be it Nanomedicine, Nano dentistry, Nano on the move, technology or rather Nano is in the air. It may sound fictitious now, but in the future, we dentists will be replaced by microscopic machines called Nano assemblers. The state of nanotechnology today represents something of a PARADOX. On one hand, the marketplace is flooded with products endorsing nanotechnology. While on the other hand, understanding of the underlying properties of Nanoscale materials and structures is still at a rudimentary level with dawning fear of singularity where Nanomachines will rule the human mind. Thus, it is rightly compared with a toy box of nature having the ability to create limitless things. It's on how we use this toy box will determine humanity's tomorrow-Good or bad.
\end{abstract}

Keywords: Nanotechnology; Nanodentistry; Nanomaterials; Nanrobots

\section{Introduction}

"NANODENTISTRY", the trending branch will soon make possible utilization of nanomaterials and nanobots for the maintenance of comprehensive oral health. Micro-technology can be defined as the building of structures through a "top-down" process of the bulk deposition and removal of a material, as occurs in a microelectronic industry [1]. Conversely, nanotechnology is the formation of a structure through a "bottom-up" process, starting at the molecular level and using atoms or molecular scale materials, which results in the desired structure [2]. In this review, we will examine not only how tightly nanotechnology tentacles have gripped dentistry as it has the potential to create many new materials and devices with wide-ranging applications but also its unaware presence around us along with its future implications, assumed hazards and specific problems faced for it to mushroom in India. Nanotechnology raises many of the same issues as with the introduction of any new technology, including concerns about the toxicity and environmental impact of nanomaterials, and their potential effects on global economics, as well as speculation about various doomsday scenarios.

\section{Nanodentistry as a Bottom up Approach}

\section{Local anaesthesia}

It is a colloidal suspension of active analgesic nanorobots instilled on the patient's gingiva. After contacting any surface, the nanorobots finally reach the pulp through dentinal tubules and hence shut down all sensitivity. Because of different tubular branching patterns, the tubular density may present a significant challenge to navigation. Hence a total path of $10 \mathrm{~mm}$ from the tooth surface to the pulp will be travelled by $100 \mu \mathrm{m} / \mathrm{second}$. The presence of natural cells that are constantly in motion around and inside the teeth including human gingival and pulpal fibroblasts, cementoblasts, odontoblasts, and bacteria inside dentinal tubules, lymphocytes within the pulp or lamina propria suggests that such journey would be feasible by cellsized nanorobots of similar mobility [3].

Advantages: Greater patient comfort, reduced anxiety as it is needleless, selective analgesic effect, reversible switchable action and reduced adverse effects.

\section{Hypersensitivity cure}

Dentin hypersensitivity is generally caused by changes in hydrodynamic pressure in the pulp and hypersensitive teeth have 8 times higher surface density and two times larger diameter of dentinal tubules compared to nonsensitive teeth. These nanorobots could selectively occlude tubules in minutes with native biological materials hence providing a quick relief to the patient though there are many therapeutic agents for this common painful condition that provide temporary relief [2].

\section{Nanorobotic dentrifice (dentifrobots)}

Mouthwash or toothpaste containing nanorobotic structures could patrol all supragingival and subgingival surfaces hence metabolizing all trapped organic matter into harmless vapours. Surprisingly these do not harm the $\sim 500$ species of the harmless oral microflora of a healthy ecosystem. They would crawl at 1-10 microns/sec and even deactivate themselves if swallowed. Hence, they prevent halitosis by preventing bacteria putrefaction. With this kind of daily dental care available from an early age, conventional tooth decay and gingival disease will disappear into the annals of dental history. Upon decay there will be a need to rinse with a sol containing nano assemblers which will swim to areas of the mouth where a cavity is formed and eliminate both bacteria and decay hence, repair the tooth by building new tooth 
structure. One can rinse ultimately to spit these tiny machines [4]. Thus, no drilling and filling will be required in the near future.

\section{Dental durability and cosmetics}

Replacing upper enamel layers with pure sapphire and diamond can improve esthetics and tooth durability by making it tougher due to the incorporated carbon nanotubes. It offers a very interesting alternative to routine whitening procedures. Though sapphire too is susceptible to acid corrosion like enamel it can be manufactured in virtually any colour of the rainbow [5].

\section{Orthodontic treatment}

These would directly manipulate the periodontal tissues allowing rapid and painless tooth straightening within minutes when injecting in periodontal tissues through the biological basis is unexplained [6].

Nanosensors in orthodontics: Lapatki and Paul developed a true scale smart bracket using a stress sensor chip. Rudolf and Fercec developed methods for measurement of forces and moments acting on a human tooth in an orthodontic system. Shi et al. developed a piezoresistive stress sensor for the measurement of orthodontic forces that act on a tooth in invisible aligners [7,8]. Thus all these developments demonstrate the feasibility of using miniaturized and ultra-thin sensors to determine the in vitro orthodontic forces which were once a dream.

Nano coated archwires: Redlich et al. coated the orthodontic wire with nickel-phosphorous film impregnated with fullerene nanoparticles of tungsten disulfide (IF-WS2). It was observed that the friction coefficient of the wire was significantly reduced by up to $65 \%$. Also, the friction forces were reduced by up to $54 \%$. Liu et al. performed a study to access the antimicrobial and osteoinductive properties of stainless steel wire coated with silver nanoparticles. The results show that stainless steel wire coated with silver nanoparticles exhibit strong bactericidal and osteoinductive properties that make it a promising pharmaceutical material in orthopaedic surgery. Zhao et al. investigated nano-hardness, wear resistance and pseudoelasticity of hafnium implanted NiTi shape memory alloy and found it to exhibit better wear resistance than the untreated NiTi in the aspects of a lower initial friction coefficient with a much longer fretting time. Also, the Hf-NiTi exhibited improved pseudoelastic behaviour and retained their surface integrity even after being strained in tension to $10 \%$. Mhaske et al. performed a study to access the anti-adherent and antibacterial properties of surface modified stainless steel and NiTi orthodontic wires with silver nanoparticles against $L$. acidophilus. The authors observed that the wires coated with silver showed an antiadherent effect against $L$. acidophilus leading to minimization of accumulation of dental plaque and, thus, the minimization of dental caries during orthodontic treatment $[7,8]$.

Fabrication of hollow wires: Hollow wires are wires coated with $\mathrm{NiTi} / \mathrm{Ni}-\mathrm{TiO} 2$ composite nanoparticles via the synthesis method called ultrasonic spray pyrolysis (USP). The precursor solution for the synthesis of spherical NiTi particles is prepared from an orthodontic wire with a chemical composition of $\mathrm{Ni}$ (amount fraction $\mathrm{x}=51.46 \%$ ) and $\mathrm{Ti}(\mathrm{x}=48.54 \%)$. A textile or polymer fibre is coated with NiTi nanoparticles via electrospinning and then the fibre is removed to produce a hollow wire for orthodontic purposes. This wire could potentially have the shape-memory and superelasticity properties, while possibly reducing the material needed for the wire production [9].
Orthodontic brackets: A new material which contained polysulfone embedded with hard alumina nanoparticles was developed in the year 2012 by UC3M for making orthodontic brackets. The material innovated had the properties of strength, reduced friction and biocompatibility while maintaining the transparency of the bracket. Orthodontic brackets have been coated with nitrogen-doped titanium dioxide. The activation of Nitrogen-doped Titanium dioxide leads to the formation of $\mathrm{OH}$. Free radicals, superoxide ions $\left(\mathrm{O}_{2}\right)$, peroxyl radicals $\left(\mathrm{HO}_{2}\right)$ and hydrogen peroxide $\left(\mathrm{H}_{2} \mathrm{O}_{2}\right)$. These chemicals, through a series of oxidation reactions, react with biological molecules such as lipids, proteins, enzymes and nucleic acids, damage biological cell structures, but also exert antimicrobial activity [9].

\section{Photosensitizers and carriers}

Quantum dots can be engineered to absorb and emit many wavelengths of light with very sharp precision. This makes them ideal for protein-protein interaction studies as they can be linked to molecules to form long-lived probes.

They can track biological events by tagging specific proteins or DNA in order to follow their progress through biological pathways. In medicine, quantum dots could be used for diagnostic purposes. They can produce exceptional images of tumour sites. Quantum dots can be effectively used to bind to the receptor on the target cell and produce reactive oxygen to kill the cell when stimulated by UV light [10].

\section{Diagnosis of oral cancer}

This can be done utilizing Nanoelectromechanical systems (NEMS), Cantilever array sensors and Multiplexing modality. NEMS Converts chemical energy into electrical signal and cantilever array sensors promise an ultrasensitive mass detection technology. While multiplexing modality helps in sensing large numbers of different biomolecules simultaneously in real time. Thus, this technology can be applied for diagnosis of oral cancer and also detection of bacteria, fungi, and viruses [10].

Oral fluid nanosensor test (OFNASET): It is a handheld, automated, easy to use an integrated system which enables simultaneous and rapid detection of multiple salivary and nucleic acid targets. It is developed by UCLA Collaborative Oral Fluid Diagnostic Research Center [10].

Optical nanobiosensor: The nano biosensor is a unique fibre optics based tools which allows the minimally invasive analysis of intracellular components such as cytochrome $c$ which is a very important protein to the process which produces cellular energy and is well known as the protein involved in apoptosis that is programmed cell death $[11,12]$.

\section{Treatment of cancer}

This can be done utilizing nano brachytherapy materials like BrachySil $^{\text {Tm }}$ (Sivida, Australia) delivering 32P which are under clinical trial. Drugs crossing the blood-brain barrier can provide more effective treatment of brain tumours, Alzheimer's and Parkinson's disease and are under development. Nanoparticles provide a unique opportunity for rapid delivery of active compounds and can be used to cross the blood-brain barrier (BBB) which represents a major barricade for a lot of chemical entities. Predominantly due to their size, nanoparticles can pass through the endothelial cell membrane Gene therapy vector which will be non-viral and nano in a structure are also promising surprising results. An assembler built repair vessel and a DNA repair 
machine are hypothetical models repairing the defective DNA and replacing it with new one thus eliminating many genetic disorders $[10,11]$.

\section{Tooth repair}

Nanodental techniques for major tooth repair may evolve through several stages of technological development, first using "genetic engineering, tissue engineering and tissue regeneration", and later growing the entire new teeth. Ultimately, the nanorobotic manufacture of a biologically autologous whole tooth replacement including both mineral and cellular components should become feasible to undertake within the time and economic constraints of an ordinary dental visit using an affordable desktop $[13,14]$.

\section{Tooth renaturalization}

Tooth renaturalization is a promising technique in the near future utilizing nanotechnology to its utmost advantage. This may help those who wish to replace their amalgam filling with native biological materials. The tooth can be remanufactured to be indistinguishable from the natural one and yet possess good strength $[13,14]$.

\section{Nanodentistry as Top-Down Approach}

\section{Nanocomposites}

These consist of nanoparticles homogeneously distributed in resins. The nanofiller is aluminosilicate powder with mean size $80 \mathrm{~nm}$, the ratio of alumina to silica is 1:4 and refractive index of 1.503 .

Advantages: Exhibits superior hardness, flexural strength, modulus of elasticity, translucency and reduced shrinkage with excellent handling properties [15].

These nanocomposites using advanced methacrylate resins and curing technologies possess both superior esthetics as well as excellent mechanical properties.

Nanofillers: These require bottom-up manufacturing approach. However, these milling procedures can reduce the size just till $100 \mathrm{~nm}$. Hence, some synthetic chemical processes were needed to be used. Thus nanosized fillers for a dental nanocomposite that is Filtek Supreme Universal Restorative (3M ESPE Dental Products) was produced. Other products included nanofiller such as Premise and Prime and Bond. Filtek Supreme is made in 4 opacities (in decreasing order of opacity); dentin, body, enamel and translucent.

Two types of nanofillers:

- Nanomeric or NM particles: Monodisperse and nonagglomerated silica nanoparticles dispersed in aqueous colloidal silica sols.

- Nanoclusters or NC particles: Spheroidal agglomerated particles with a size of 2 to $20 \mathrm{~nm}$ of zirconium silica particles with 3methacryloxypropyltrimethoxysilane as a coupling agent and resin system of bisphenol A, glycidyl di-methacrylate, tri-ethylene glycol di-methacrylate.

\section{How do NC particles differ from hybrid filler particles?}

Hybrid fillers (average size $1 \mu \mathrm{m}$ ) particles cannot be further subdivided. However, nanoparticles can be broken down further providing smaller defects and hence better retention. When a mismatch in refractive index exists between the filler particles and resin the particles will scatter light and produce opaque materials. In NM-particle materials, the size of the particles is far below the wavelength of light. When light strikes, long-wavelength light passes directly through hence materials exhibit high translucency. Thus a very low opacity Nanocomposite provides a vast range of shade and opacity options [16].

\section{Nano bonding systems}

Traditionally, three steps of etching, priming and bonding were required but the all-in-one system has integrated them into the "onestep" system eg. the G-Bond one-bottle launched by GC Corporation. It decalcifies the surface of the dentin only slightly with no exposure of collagen fibres. Thus an extremely thin interface is formed wherein functional monomers react with hydroxyapatite at the "nano" level, to form insoluble calcium. This interface is more durable than that formed by other bonding materials. It is aptly termed as the Nano Interaction Zone [17].

Advantages: Higher dentin bond strength and no shaking of bottle required since the nanoparticles are stable with ensured homogeneity.

Indications: All direct, light-cured bonding procedures, indirect bonding procedures when combined with $3 \mathrm{M}^{\mathrm{sm}}$ ESPE $^{\mathrm{m}}$ RelyX $\mathrm{X}^{\mathrm{sw}}$ ARC, Root surface desensitization, porcelain and composite repair, bonding veneers in combination with RelyX ${ }^{\mathrm{Tw}}$ Veneer Cement from 3M ESPE.

Available in two delivery options:

- Easy to use unit dose

- Convenient squeeze bottle delivery with attached "pinch and flip" cap for less mess and less waste.

Advanced bonding systems: Development of adhesive materials will probably involve biomimetic approaches, adopting mechanisms found in living organisms. Research efforts have already been fruitful in developing bonding mechanism for both dry and wet substrates. Dry bonding has copied the ability of animals such as geckos to suspend their bodies from surfaces by the retentive forces of a network of fibrils attached to their feet. They were then successful in formulating a network of carbon nanotubes, grown on silicon substitutes, embedded in a polymer matrix, which resembled the foot hair morphology of the animal. Implications - bracket base $[7,18]$.

Nanosolutions: Produce unique and dispersible nanoparticles, used in bonding agents. Ensures homogeneity and that the adhesive is perfectly mixed every time. Available with the trade name: Adper O, Single Bond Plus, Adhesive Single Bond $[7,16]$.

\section{Impression materials}

Nanotech Elite $\mathrm{H}_{-} \mathrm{D}^{\star}$ from company Zhermack is available with nanotechnology application. This consist of nanofillers admixed with vinylpolysiloxanes hence produce a unique addition siloxane impression material.

Advantages of nanotech elite $\mathrm{H}-\mathrm{D}^{*}$ : Better flow, improved hydrophilic properties hence fewer voids, better model pouring and enhanced detail precision [9].

Light-curing nano glass ionomer restorative

$\operatorname{Ketac}^{\mathrm{Tm}}$ Nano Light Curing Glass Ionomer.

Clicker $^{\mathrm{mit}}$ Dispenser - mix the perfect dose every time faster- with less mess. 
Meets a Wide Range of Clinical Indications such as deciduous teeth restorations, transitional restorations, small Class I, III and V restorations, sandwich restorations, and Core build-ups with superb polish and excellent esthetics. In vitro testing results show Ketac Nano restorative has higher initial polish than other glass ionomer restorative materials. Shades of Ketac Nano restorative were developed to match the shade targets of Filtek ${ }^{\mathrm{mi}}$ Supreme Plus Universal Restorative. Ketac ${ }^{\mathrm{Tm}}$ Nano Light-Curing Glass Ionomer Restorative shows high fluoride release that is rechargeable after being exposed to a topical fluoride source. Additionally, in-vitro tests showed Ketac Nano restorative's ability to create a caries inhibition zone after acid exposure [19].

\section{Nanoadhesive-Poss}

Polyhedral oligomeric Silse Sioux (Poss) enables the design of additives that make plastics that are unusually lightweight, durable, heat tolerant and environment-friendly. Poss combines organic and inorganic materials in molecules with an average diameter of 1.5 nanometers. They can be used as either additives or replacements for traditional plastics. Current applications of Poss include dental adhesives in which a strength resin provides a strong interface between the teeth and the restorative material. In addition, tests have shown that Poss materials are much more resistant to radiation damage and erosion than convention polymers [12].

\section{Nanoporous silica filled composite}

Nanoporous silica filled composite is a fairly new material still in experimental form, proven to increase wear resistance in posterior applications Nano-sized porous silica fillers allow the monomer to interpenetrate it, through a capillary force; the monomer is drawn in and out of the filler, reinforcing the composite and increasing the durability of the bowing between the two phases. By impregnating organic monomer into the pores and adding a light cure system, a solid organic/inorganic nanostructure is formed [12].

\section{Denture teeth}

Denture tooth, made of nanocomposite resin, has recently been developed as a highly polishable, stain and impact resistant material, consisting of a comonomer of ethanure dimethacrylate (UDMA) and methacrylate (MMA) and uniformly dispersed nanosized filler particles. It can be speculated that these teeth possess better surface hardness compared to conventional acrylic and micro filled teeth $[20,21]$.

\section{Nano implants and bone replacement materials}

$3 \mathrm{i}$ introduced its NanoTite implant. The use of nano-scaled calcium phosphate $(\mathrm{CaP})$ crystals applied to the surface of the implant has been shown in pre-clinical trials to facilitate the bond between bone and implant. 3i applied this nanotechnology to its already successful Osseotite implant resulting in the NanoTite. CaP is biologically compatible with bone and tissue - its use may reduce the risk of implant failure.

Advantages: Substantial improvement in the rate and extent of osseointegration has been noted, a marked increase in bone-to-implant contact and improved bone fixation which may lead to greater stability for the implant.
Some hydroxyapatite nanoparticles are also being used to treat bone defects such as Ostim ${ }^{\odot}$ (Osartis GmbH, Germany) HA, VITOSS (Orthovita, Inc., USA) HA + TCP, NanOss ${ }^{\text {Tit }}$ (Angstrom Medica, USA) HA $[21,22]$.

\section{Nanoneedles and nano tweezers}

These needles being of small size hardly make the patient feel the prick hence they render painless injections. These utilize nano-sized stainless steel crystals in them. They are available with Trade name of Sandvik Bioline, RK 91 ${ }^{\mathrm{ma}}$ needles [AB Sandvik, Sweden]. While Nanotweezers are still under development which endeavors to make cell surgery possible [22].

\section{Nanox}

It's the revolution of a portable toothbrush with an automatic refill of paste. It's a one-touch portable toothbrush with built-in toothpaste. Toothpaste and toothbrush are joined together having a built-in toothbrush. It has proven its top quality worldwide with a smooth onetouch manner. Upon pushing the button twice or thrice according to the volume required, an adequate volume of toothpaste comes out smeared with the brush. The design based on mouth human body engineering, even molar tooth is easily gargled without giving much strength to Northridge. Being an airless vacuum pump, toothpaste could be maintained freshly for a long time. It is assumed to be very clean, because of no clogging or leakage. It's highly advantageous while travelling, during a stay at hotels and resorts or even during outdoor activities [23].

\section{Nano and the Future}

\section{Foglets}

It's a hypothetical microscopic robot which has a body about the size of a human cell with 12 arms radiating in different directions. A cluster of these might form a 'robot crystal by connecting with each other in a lattice structure.Thus if left in the air, these robots can rightly be called Foglets forming Utility Fog, which may have many useful medical applications [24].

\section{Tiny tech}

These diagnostic machines promise to travel and repair the cancerous cells in the bloodstream [24].

\section{Neuron replacer and brain cell enhancer}

A hypothetical Nanorobot at work in the human brain, replacing or augmenting human nerve cells with artificial (Nanorobotic) nerve cells. The blue, octopus-like nanobot is one of the billions of brain cell enhancers. The central sphere houses a computer, with a storehouse of information equal to many large libraries. Billions of spheres are in contact with one another, as well as with the brain cells, creating a secondary gestalt mind that interfaces with the brain's original gestalt mind [24].

\section{Personal nanomedical appliance to replace blood}

Includes Vasculoid, Respirocyte (artificial red blood cells), Vasculocyte, clottocyte (artificial platelet) and microbiovare (artificial phagocyte) [24]. 


\section{Primo 3M}

Primo $3 \mathrm{M}+$ is a net art 3D future physique design whose message reflects the goal of super longevity in a tongue-in-cheek advertisement for a future body. Designed with functionality in mind, its architecture is based on nanotechnology and AI. It features a meta brain with nanotech data storage system and error correction device with instant data replay and feedback. It proposes network sonar sensors map data onto the visual field and an increased frequency range with parabolic hearing. It instils in vivo fibreoptic communications backbone, an internal whole-body navigational grid, replacement organs and solar protected skin with tone texture changeability. It exhibits turbocharged suspension flexibility and biosensors which get stimulated on atmospheric tensions [24].

\section{Beyond human}

In this hypothetical model, implanted nanocomputers interface with the myriad synapses of the neurons of the brain and thus go beyond the human brain intelligence to understand [24].

\section{Wings}

In this hypothetical nanobot model, a thin sheath of nanomachinery takes man safely through space with wings of solar sails providing energy and movement [24].

\section{Resurrect}

These hypothetical nanobots wish to recreate what time has wiped out by building from fragments of DNA and computing from DNA of living relatives [24].

The Diamond Age by Neal Stephenson highlights the world of the relatively near future. "The Feed" allows most anything to be created at any outlet (think Star Trek replicators), creating a minimum standard of living for all mankind through the spread of nanotechnology.

\section{Nano cosmetics including buzzsaw bots and flying saucer bots}

They are hypothetical nanobots enabling different haircuts on demand. On the other hand, Dermal display and cosmobots are hypothetical several billion dermal display nanorobots residing in the epidermis of a human hand which can be used as display screens to access real-time physiological information or other stored data. Cosmobots wait hidden in the skin to disperse the pigments from their stores as programmed by their owners - an alternative to drugstore makeup products and the time required to apply them daily [24].

\section{Wormobots and antobots}

These artificial worms aim to mine the soil and convert its waste to organic nutrients. Thus promising to be extremely useful for our farmers [24].

\section{Challenges Faced by Nanodentistry}

The various challenges faced by Nano dentistry include the difficulty in precise molecular positioning due to the small size of nanoparticles, higher expenses of mass production technique of Nanorobots, their subsequent biological compatibility issues, coordinating independent robot activities which demands high skill, large labour and heavy expenses and last but not the least the challenge of its social acceptance following ethical guidelines while keeping in mind public safety. Specifically, in India, the problems extend to slow strategic decision making, lack of funds, minimum private enterprises involvement and loss of trained manpower [25].

\section{Hazards of Nanotechnology}

\section{Biological concerns}

Nanoparticles can become widely disseminated via the bloodstream. We have already established that nanoparticles can reach in the brain, the human being's best-protected organ. Since nanoparticles are not restrained by any tissue barriers, it could be possible for them to gain access to a foetus via the placental barrier, as well. These Nanorobots, when implanted inside the body, can lead to carcinogenesis (C), mutagenesis $(\mathrm{M})$ and teratogenicity $(\mathrm{T})$ etc. Carbon nanotubes have been found to cause free radical formation and depletion of antioxidants in cells, pulmonary fibrosis and cancer similar to the effect of asbestos [26]. Quantum dots are made of a CdSe core with a $\mathrm{ZnS}$ shell, and all three metals are known to be toxic to different extents. They can serve as weapons for nano-bio-warfare. Unlike nuclear technology, the capacity to create biological weapons of mass destruction requires far less capital investment and is much easier to conceal [27]. Moreover, Nanobiology will enable people to live longer and have healthier lives. Longer average lifetimes will mean more people on Earth. But how many more people can the Earth sustain? Increased artificial intelligence will eventually become superior to ours, which may act as a threat. Also in advent use of these nanodevices which might get out of control can self-replicate endlessly eventually consuming all matter forming a "GREY GOO".This living fog consisting of replicating nanorobots is speculated to transform every single molecule on earth into a perfect copy of itself [28].

\section{Safety issues}

Labelling the products utilizing nanotechnology must be made compulsory. Unnecessary advertising even if their product does not contain nanoparticles must be punishable. Private companies must be encouraged to disclose the results of safety testing to the Food and drug administration [29].

\section{Conclusion}

Thus, the greatness of this technology lies in its nano-ness. The visions described above may sound unlikely, implausible, or even heretic. Yet, the theoretical and applied research to turn them into reality is progressing rapidly [30].

"Scientific devices that are dwarfed by dust mites may one day be capable of grand biomedical miracles".

\section{Acknowledgement}

It is my pleasant privilege and honour to express my sincere and heartfelt gratitude towards Dr.Manish Goyal and Dr.Akshita Sharma for their selfless support throughout.

\section{References}

1. Singh R (2012) Nanotechnology in dentistry: current achievements and prospects. JOFS 5: 9-14. 
Citation: Chawla R, Shetty KS, Prakash AT, Rathore APS, Saroch S (2018) Nanotechnology Engulfing Dentistry. J Nanosci Curr Res 3: 121. doi:

Page 6 of 6

2. Phoenix C (2007) Nanotechnology from 1959-2029. Metaverse.

3. Rajan SS, Acharya SR, Saraswathi V (2013) Nanodentistry. Indian J Sci Res 4: 233-238.

4. Shetty NJ (2013) Nanorobots: Future in dentistry. Saudi Dent J 25: 49-52.

5. Kaur J, Kaur K, Aggarwal G, Kumar H (2016) Nanotechnology in dentistry. J Pharm Sci Exp Pharmacol 5: 570-582.

6. Vishwanath A (2013) Nanotechnology-Coming soon to orthodontics and general dentistry. Vydehi Institute of dental sciences and research centre.

7. Umalkar DG, Jawale BA, Patil S (2017) Review application of nanotechnology in orthodontics: a critical review. IJCO 1: 9-12.

8. Syed SS, Kulkarni D, Todkar R, Bagul RS, Parekh K, et al. (2015) A novel method of coating orthodontic archwires with nanoparticles. JIOH 7: 30-33.

9. Panchali B, Anam M, Jahirul M, Meryam SR, Ragini M (2016) Nanoparticles and their applications in orthodontics. Adv Dent Oral Health 2: 555-584.

10. Kumar S (2010) A critical review of the implication of nanotechnology in modern dental practice. National J Maxillofacial Surgery 1: 41-44.

11. Kumar S (2012) Nanotechnology: The emerging science in dentistry. JOFR 2: 33-36.

12. Chandra MPE, Kumar SM, Parthiban S (2012) Nanotechnology in dentistry - a review. Int J Biol Med Res 3: 1550-1553.

13. Rajanandhini S, Lavanya B, Chellam R (2014) Nanorobotics in dentistry. Int J Adv Eng Res 8: 254-56.

14. Das A, Nasim I (2017) Nanotechnology in dentistry: a review. J Adv Pharm Edu Res 7: 43-45.

15. Mitra SB, Wu D, Holmes BN (2003) An application of nanotechnology: in advanced dental materials. JADA 134: 205-208.

16. Sharan J, Singh S, Lale SV, Mishra M, Koul V, et al. (2017) Applications of nanomaterials in dental science: a review. J Nanosci Nanotechnol 17: 2235-2255.
17. Bishara SE, Ajlouni R, Soliman MM, Oonsombat C, Laffoon JF, et al. (2007) Evaluation of a new nano-filled restorative material for bonding orthodontic brackets. World J Orthod 8: 8-12.

18. Bhardwaj A, Bhardwaj A, Misuriya A, Maroli S, Manjula S, et al. (2014) Nanotechnology in dentistry: present and future. J Int Oral Health 6: 121-126.

19. (2014) Nanomaterials for Industry 2014.

20. Suzuki S (2004) In vitro wear of nano-composite denture teeth. J Prosthodont 13: 238-243.

21. Aeran H, Kumar V, Uniyal S, Tanwer P (2015) Nanodentistry: is just a fiction or future. J Oral Biol Craniofac Res 5: 207-211.

22. Awan KH, Khan S (2014) Nanotechnology and its role in dentistry: a review. Hard Tissue 3: 10-14.

23. Pradhan D, Sharma L, Chaudri A (2017) Nanotechnology: future of dentistry. Int J Oral Health Med Res 3: 134-136.

24. Foresight Institute (2014) Advancing beneficial nanotechnology, nano medicine art gallery.

25. Kennedy J (2008) Nanotechnology: the future is coming sooner than you think.

26. Ramesh P (2011) Nanotechnology: application, nano hazard and its toxic effects. EJLS 1: 30-38.

27. Pugiliese G (2008) Nanotechnology: Cancer risk.

28. Shashirekha G, Jena A, Mohapatra S (2017) Nanotechnology in dentistry: clinical applications, benefits, and hazards. Compend Contin Educ Dent 38: e1-e4.

29. (2013) Nanotechnology just as big a threat as GMO needing desperate labelling.

30. Dalai DR, Bhaskar DJ, Agali CR, Singh N, Gupta D, et al. (2014) Futuristic application of nano-robots in dentistry. Int J Adv Health Sci 1: 16-20. 